\title{
Propuesta de una estrategia de comunicación para la empresa hotelera Gaviota
}

DOI: https://doi.org/10.33262/ap.v1i2.6

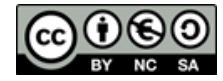

Proposal of a communication strategy for the hotel company Gaviota

Dayana Pulido de la Paz. ${ }^{1} \&$ Yasser Vázquez Alfonso. ${ }^{2}$

\begin{abstract}
.
The peak of the social nets of Internet (RSI) they have impacted in a positive way the strategy of marketing of the tourist companies. The present work intends to elaborate a communication strategy for the use of social nets in the company hotel Gaviota. To arrive to this result, he was carried out a diagnosis that I show a group of inadequacies in the use of the social nets in the company hotel Gaviota. In particular, from the theory of the communication and the foundations of the use of the social nets, the proposed strategy was designed, as a result scientific that completed in turn with the current requirements of the methodology of the tourist investigation. In this sense, he intends the use of social nets from the elaborated strategy that offers an answer theoretical-practice to the challenges of the Cuban hotel companies, especially given in the current dynamism propitiated by the process of changes of the Cuban tourist sector. The strategy in the theoretical thing contributes a group of ideas rectors that guides the directive of the company hotel Gaviota a to proceed for the effective use of the social nets in the promotion of the products and services of the company. The feasibility of the same one was determined using the consultation to experts.
\end{abstract}

Keywords: Communication strategy, Gaviota, Social Nets of Internet.

\section{Resumen.}

\footnotetext{
${ }^{1}$ Universidad de La Habana. La Habana, Cuba. dayanapp@ftur.uh.cu

${ }^{2}$ Universidad de La Habana. La Habana, Cuba. yalfos1@gmail.com
} 
El auge de las redes sociales de Internet (RSI) han impactado de forma positiva la estrategia de marketing de las empresas turísticas. El presente trabajo se propone elaborar una estrategia de comunicación para el uso de redes sociales en la empresa hotelera Gaviota. Para arribar a este resultado se realizó un diagnóstico que develó un conjunto de insuficiencias en el uso de las redes sociales en la empresa hotelera Gaviota. En particular desde la teoría de la comunicación y los fundamentos del uso de las redes sociales, se diseñó la estrategia propuesta, como resultado científico que cumplió a su vez con los requisitos actuales de la metodología de la investigación turística. En este sentido se propone el uso de redes sociales desde la estrategia elaborada, que ofrece una respuesta teórico-práctica a los retos de las empresas hoteleras cubanas, especialmente dado en el actual dinamismo propiciado por el proceso de cambios del sector turístico cubano. La estrategia en lo teórico aporta un conjunto de ideas rectoras que orienta a los directivos de la empresa hotelera Gaviota un proceder para la utilización eficaz de las redes sociales en la promoción de los productos y servicios de la empresa. La factibilidad de la misma fue determinada utilizando la consulta a expertos.

Palabras claves: Estrategia de comunicación, Gaviota, Redes Sociales de Internet.

\section{Introducción.}

El turismo y el sector de los viajes se ha visto transformado en la última década influenciado por los cambios socioeconómicos y por el acelerado desarrollo de las Tecnologías de la Información y las Comunicaciones (TICs). Hoy, es considerado por la Organización Mundial del Turismo como uno de los principales actores del comercio internacional y una de las principales fuentes de ingresos para numerosos países en desarrollo. En tal efecto, favorece la generación de empleos e impulsa otros sectores a los que se relaciona como la construcción, la industria agropecuaria, telecomunicaciones y otros.

La realidad actual del turismo se muestra con una tasa de crecimiento promedio anual de 4.5

$\%$ durante los últimos 50 años y en relación a los ingresos representa 7\% del total de exportaciones mundiales y más del 35\% de las exportaciones de servicios (OMT, 2016). El escenario turístico actual se presenta con un nuevo modelo basado en la innovación, tecnología, sostenibilidad y accesibilidad; con un perfil del viajero diferente y una oferta turística cambiante al nuevo contexto social, económico y cultural. Mientras que en la esfera de los negocios se han introducido nuevos patrones acorde a los canales y maneras modernas de llegar a los clientes; de igual forma los destinos han encontrado alternativas de conectar con el escenario que se presenta. Ante este escenario las redes sociales de Internet han llegado al sector turístico para quedarse como una forma de conectar a cientos de personas de forma rápida y cómoda.

El auge de las redes sociales de Internet, o simplemente redes sociales, son plataformas digitales que ha impactado de forma positiva de la estrategia de marketing de las empresas, 
sea cual sea el sector al que pertenezcan. Sin embargo, para las empresas turísticas es aún mayor pues son empleadas en todos los momentos del viaje turístico. Desde esta perspectiva, las redes son empleadas por los viajeros en la planificación, realización del viaje; así como una vez finalizado el viaje. Esto sucede porque dichas plataformas permiten a los usuarios conocer y estudiar las ofertas; realizar reservas, compras y compartir estados de opinión y satisfacción de clientes (García, Cortés, \& Galmés, 2015).

Desde otro punto de vista, las redes sociales de Internet representan una herramienta de marketing y un nuevo escenario para los negocios; pues a través de estas las empresas buscan aumentar su mercado, fidelizar clientes, contactar socios y expandir su presencia en el mundo. Las comunidades virtuales que constituyen estas redes son espacios de comunicación influyentes para posicionar las marcas de las empresas, productos y servicios.

En estas las empresas exponen sus ofertas, facilitan canales de pago, ofrecen información; además son necesarias en la implementación de las estrategias de comunicación para acercarse al mercado y buscar nuevas oportunidades de negocio (Miranda \& Cruz, 2016). En este contexto, constituye un reto para las organizaciones porque es difícil encontrar una plataforma integrada que permita a las empresas conectarse con sus clientes y al mismo tiempo mejorar su experiencia (Grandi, 2013). Además, se enfrentan al desafío del acelerado ritmo de los avances tecnológicos. Sin embargo, todo esfuerzo realizado por perfeccionar el uso de estas redes es válido y necesario pues influyen de manera incisiva en la reputación de la instalación considerando que más del $50 \%$ de los viajeros comparten alguna foto u opinión relacionada con su experiencia (SGM, 2016).

En el entorno de los establecimientos hoteleros, la penetración de las RSI fue tardía en relación a otros sectores como la aviación y las agencias viajes (García, Cortés, \& Galmés, 2015); sin embargo, hoy son instrumentos efectivos involucrados en la marcha de los planes de comunicación de las empresas turísticas. Esto ha provocado un retraso en cuanto a las facilidades y funcionalidades disponibles para los usuarios en estas plataformas, pues una gran parte de los hoteles en el mundo, no aprovechan al máximo las bondades que brindan los medios sociales de Internet para la comercialización de sus productos y servicios (Miranda \& Cruz, 2016).

De forma general las RSI deben ser empleadas por las empresas las emplean como una forma de comunicación y promoción directa de servicios (Mena, 2013); pero además debe contener facilidades que permitan establecer un lazo más fuerte entre la organización y el cliente. Es por ello que actualmente las empresas exitosas insertadas en las RSI ofrecen las facilidades a los usuarios no solo de conocer y estudiar las ofertas; sino también realizar reservas, compras y compartir estados de opinión y satisfacción de clientes (García, Cortés, \& Galmés, 2015); aprovechando toda la información generada para mejorar la experiencia del turista. 
En el ámbito turístico cubano, la reciente inserción de las empresas en la web ha supuesto un cambio paradigmático en la forma en que tiene lugar el proceso de comunicación entre las empresas y sus públicos. Cuba ha reconocido la importancia que tiene Internet en el desarrollo de la oferta turística cubana, por ello forma parte de la estrategia de comunicación de la marca país (Villasante, 2010). Sin embargo, la oferta de alojamiento turístico cubano presenta limitaciones en este sentido.

Las organizaciones de alojamiento se encuentran dispersas y con poca presencia en las Redes Sociales de Internet, tienen problemas su la infraestructura tecnológica que, a su vez limita la conectividad. Otras limitaciones radican en el desinterés por el tema y falta de conocimiento sobre el uso de estas. Además, se puede señalar que no se aprovecha eficientemente el uso de las Redes Sociales para la comercialización de la oferta hotelera cubana y por consiguiente esto repercute en los resultados finales de dichas organizaciones.

Las políticas sobre desarrollo turístico cubano plasmadas en los Lineamientos del Partido Comunista de Cuba en el 2014, abogan por el perfeccionamiento de las formas de comercialización y de comunicación promocional utilizando las Tecnologías de la Información y las Comunicaciones y precisando una adecuada administración de los recursos tecnológicos (PCC, 2014).

Gaviota Hoteles es la empresa hotelera cubana que mejor emplea sus redes sociales; sin embargo, se detectaron irregularidades en el uso de las redes sociales de Internet que ponen en evidencia el desaprovechamiento de estas plataformas digitales. (Ruiz, 2018). Ello se debe a que el equipo de gestión de RSI y del departamento de Promoción y Mercadotecnia en general utilizan diferentes cuentas en redes sociales como Facebook, Yourtube, Google Plus y Tiwtter de forma espontánea y dispersa. Ante esta situación se plantea el siguiente objetivo proponer una estrategia de comunicación de redes sociales de Internet que permita la promoción de los productos y servicios para el grupo hotelero Gaviota.

\section{Materiales y métodos.}

El presente estudio es de tipo exploratorio debido a que se identificaron las principales barreras de la comunicación organizacional en el ámbito de las Redes Sociales. En este sentido, se realizó un acercamiento al tema en este nivel de investigación lo que permite vislumbrar un panorama actual sobre las limitaciones que presentan los grupos hoteleros cubanos a través de las redes sociales como canal de comunicación.

La investigación desarrollada se concibe en dos fases, la primera tuvo como objetivo principal la determinación del problema de investigación, la realización de un amplio análisis del estado de arte a través de la consulta bibliográfica de variadas fuentes. La segunda consistió en la elaboración de la propuesta de estrategia de comunicación a través de las redes sociales para el grupo hotelero Gaviota. 
Es un estudio de tipo descriptivo, con un carácter empírico. Por otra parte, el estudio es no experimental, pues no se concentra en manipular las variables para comprobar cambios en las mismas; este tipo de investigación se llevan a cabo para poder observar el fenómeno tal y como se presenta para poder analizarse en su contexto natural. ya que no se manipulará de forma deliberada las variables. Teniendo en cuenta los resultados, la investigación es mixta (cualitativa y cuantitativa).

Para la ejecución de la investigación, se tomó como población el total de trabajadores que integran los departamentos de comunicación y comercial de las instalaciones de alojamiento pertenecientes a la empresa Gaviota. De ahí que, se conformaron dos muestras; la primera para las entrevistas, conformándose de forma intencional, considerándose los empleados vinculados directamente al proceso de comunicación a través de las RSI y teniendo en cuenta los siguientes criterios: cargo que ocupa, experiencia laboral, trabajo con las redes sociales de Internet, años de trabajo en la instalación (mínimo 1 año vinculado al departamento), siendo el muestreo de tipo intencional puro y la muestra no probabilístico; la segunda muestra se escogió para las encuestas, teniendo en cuenta la misma población, siendo este muestreo de tipo aleatorio y la muestra probabilística.

\section{Métodos teóricos.}

El primer método recurrido desde el principio y durante la investigación fue la revisión bibliográfica. Esta permite el análisis teórico existente a nivel internacional del empleo de las Redes Sociales de Internet, y una panorámica del empleo de las mismas, la Web 2.0 y las nuevas tecnologías de información y la comunicación (NTICs) a nivel nacional e internacional.

Para el trabajo se consideraron artículos de revistas científicas (Journal of Communication, CULCyT, Revista de Comunicación Vivat Academia, Scielo, etc.), revistas especializadas en los temas de comunicación y turismo (Hosteltur, Rotary, Chasqui, Excelencias, Periódico Granma, Cubadebate, Periódico El Político), blogs especializados en el tema de la gestión de redes sociales (IAB, Marketing SGM), informes de las organizaciones estudiadas y libros sobre la Comunicación Organizacional (Manual de Comunicación Organizacional para empresas y organizaciones, de la Gestión de la Comunicación en las Organizaciones, Manejo de la Comunicación Organizacional).

Como una limitación, se debe mencionar que no se pudo acceder a toda la información que se encuentra en las bases de datos, debido a la restricción o bien al formato de presentación de algunos artículos; así como no se contó con la cooperación del Grupo Hotelero Gaviota para formar parte del estudio.

Se llevó a cabo una revisión documental de documentos oficiales de la organización que permitió identificar rasgos principales que caracterizan la gestión de la comunicación en los grupos hoteleros estudiados. 
El método histórico-lógico permitió sistematizar los conocimientos relacionados con el tema tratado, yendo de lo más general a lo más particular, a partir de la lectura de diversas investigaciones que abordan resultados reales y actuales relacionados con el uso de las redes sociales. También se empleó para caracterizar la trayectoria de los diferentes grupos en el sector turístico cubano.

Se emplearon cuatro métodos: la entrevista, la observación y la encuesta. La entrevista en profundidad fue una herramienta muy importante de obtención de la información y las encuestas semiestructuradas son técnicas muy importantes empleadas en la investigación. La observación permitió identificar las principales cualidades en el uso de las Redes sociales en las organizaciones.

\section{Resultados y discusión.}

\section{Concepción de la estrategia.}

La estrategia consta de 4 fases, cada una de estas utilizan un conjunto de métodos y técnicas que permiten realizar las estimaciones y valoraciones respecto a lo temas relacionado con el objeto de estudio. Considerando las principales limitaciones y fortalezas detectadas en el diagnóstico, Gaviota presenta insuficiencias en su posicionamiento; por lo que consecuente, el tipo de estrategia completar.

Asumiendo estos criterios, se la estrategia de comunicación en las Redes Sociales de Internet para el grupo hotelero Gaviota comprende una serie de acciones reguladas por fundamentos teóricos que explican cómo proceder para propiciar el desarrollo de la promoción de los servicios y productos turísticos del grupo Gaviota.

La concepción de la estrategia está sobre la base del pensamiento dialéctico-materialista, lo que asume los nuevos conocimientos solucionan los problemas de la práctica. Así mismo, parte del método materialista-dialéctico, que favorece las vías para la interpretación de la realidad objetiva de la naturaleza y la sociedad. En ese orden de ideas cabe señalar que, la estrategia que se propone concibe la participación activa del sujeto dentro del proceso comunicativo en el ámbito de las redes sociales.

La utilidad de la estrategia que se propone, es el resultado de un proceso de investigación científica, que radica precisamente en la concepción teórica que la respalda y en la necesidad de solucionar con eficacia las dificultades que se manifiestan en el uso de las redes sociales por parte del Grupo. En este sentido, la estrategia se estructura en componentes interrelacionados en función del objetivo trazado (ver a figura 1).

Figura 1. Esquema de la estrategia de comunicación en redes sociales de Internet para el grupo hotelero Gaviota. 


\section{Estrategia de comunicación de redes sociales de Internet para el grupo hotelero Gaviota}

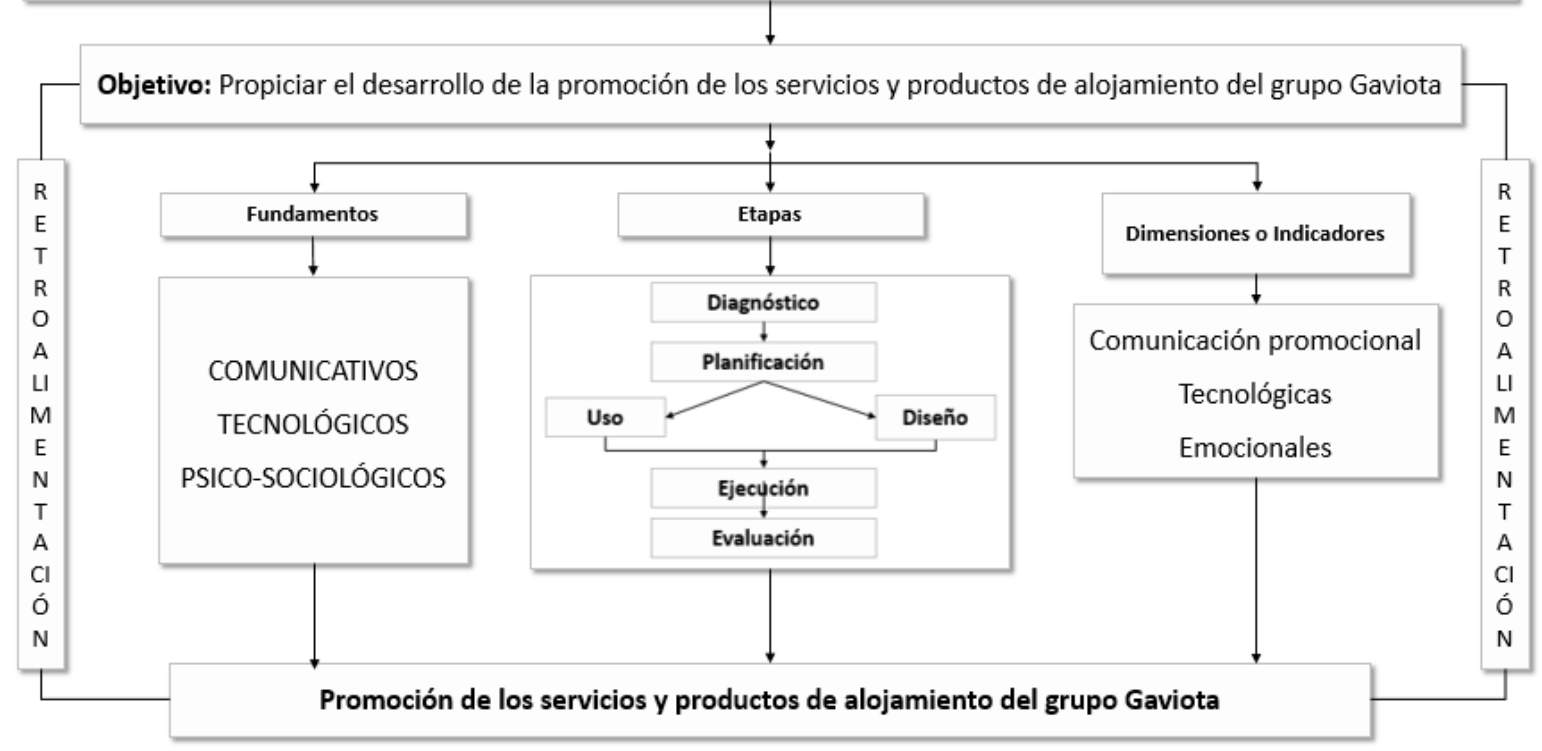

Fuente: Elaboración Propia.

Estructura y contenidos de la estrategia.

\section{Objetivo General.}

Propiciar el desarrollo de la promoción de los servicios y productos de alojamiento del grupo Gaviota.

\section{Fundamentos teóricos.}

Se tienen en cuenta como fundamentos a los aquellos elementos internos que determinan la dinámica y composición de la estrategia. Estos fundamentos son: comunicativos, tecnológicos y psicológicos. Las convergencias entre estos van a incidir en las etapas de la estrategia.

Desde el punto de vista comunicativo, la estrategia se enfoca en los procesos comunicativos que promuevan los servicios y productos del Grupo. Está sobre la base de las directrices de comunicación establecidas por la gerencia de la organización. La estrategia está orientada al contenido, siempre partiendo desde las características de los principales mercados. En este sentido las redes sociales de Internet serán aprovechadas para proyectar valores, creencias, intereses del Grupo, tratando siempre de promover los productos y servicios que conforman la oferta hotelera. Este precepto traza una línea transversal en los supuestos que definen las estrategias de visibilidad, confianza y posicionamiento. Entonces, la estrategia propuesta queda en una posición intermedia, otorgando así las principales ventajas de cada una de los tipos de estrategias globales anterior mente mencionados. 
En este sentido, la estrategia procura alcanzar una mayor visibilidad de la marca a través de la proyección adecuada, creativa y veraz de los atributos y valores asociados a la empresa; partiendo de las características de los públicos y las condiciones reales de la empresa.

Los fundamentos tecnológicos de la estrategia parten del espacio o medio por el cual se desea promocionar los productos y servicios del Grupo que son las redes Sociales de Internet. Cabe aclarar que las acciones propuestas están pensadas para las principales redes que emplea el Grupo Facebook, Twitter, YouTube y Google Plus. Se tienen como base las tecnologías multimedia para el proceso de comunicación y promoción de los servicios y productos de alojamiento. También implica el conocimiento y uso de las plataformas. En este fundamento, es un pilar concebir las herramientas de estas plataformas para facilitar la generación de conocimiento y estudios.

Los fundamentos psico-sociológicos de la estrategia de comunicación parten de que el proceso de comunicación está determinado por un emisor y un receptor condicionados por factores psicológicos y sociológicos. En el proceso de comunicación actual el receptor es también emisor y creador de mensajes y la descodificación/codificación de la información están sujetas a factores emocionales (Bono \& Abad, 2010).

Un nuevo papel de la emoción y de la memoria en el proceso publicitario y la necesidad de aplicarlo a los diferentes métodos de la investigación con consumidores, quiénes en mayor o menor medida juzgan las creatividades y el papel de las marcas. En ese sentido, se hace imprescindible complementar las técnicas de investigación tradicionales con técnicas neurocientíficas si se describen con precisión el papel de la emoción y la memoria.

Etapas, objetivos específicos y acciones de la estrategia.

Etapa

Diagnóstico
Objetivo de la etapa

Identificar las principales insuficiencias en el uso de las redes sociales en el grupo Gaviota

\section{Objetivos específicos.}

- Determinar los principales factores externos que influyen en el estado actual del uso de las redes sociales de Internet del Grupo Gaviota.

- Diagnosticar el uso de las RSI en el grupo hotelero Gaviota

Principales acciones de la etapa. 
- Determinar la situación actual sobre el uso de las redes sociales de Internet de interés para la comunicación promocional del Grupo.

- Caracterizar la oferta de alojamiento del grupo, para así conocer los principales elementos identitario.

- Explorar las condiciones organizativas y tecnológicas existentes en la organización.

- Aplicar un diagnóstico inicial mediante cuestionario, encuesta, entrevista, intercambio de experiencias, revisión de documentos y observación de actividades para la determinación de las principales deficiencias en el uso de las redes sociales.

- Triangular los resultados que posibiliten la identificación de las deficiencias y las potencialidades del uso de las redes estudiadas.

\section{Descripción de la etapa.}

Las acciones y los objetivos específicos de esta etapa deberán ser objeto de análisis, reflexión, debate y control, por los directivos en conjunto con los especialistas de comunicación del grupo. Deberán emplearse cada uno de los instrumentos elaborados para la realización de las indagaciones empíricas, se debe caracterizar por una marcada profesionalidad y flexibilidad por parte del investigador. Varios de los instrumentos diseñados para la realización del diagnóstico recogen información de diferentes indicadores y deben cruzarse las informaciones obtenidas por cada uno de ellos, con el objetivo de determinar la existencia de cualquier contradicción y las causas que la originan, así como la realización de nuevas indagaciones al respecto, si se considera necesario por parte del investigador.

Para el diagnóstico de esta estrategia se parte del análisis del entorno externo e interno de la organización. Se parte de una panorámica sobre el uso de las redes sociales en el sector turístico cubano y en particular en el ámbito hotelero. Posteriormente se profundiza en el análisis de sobre el uso de las redes sociales en el grupo Gaviota; de aquí se obtiene información válida que permite reflejar como es el empleo de las redes sociales, con especial énfasis en detectar insuficiencias.

Es importante para esta fase considerar las principales tendencias sobre el uso de las redes sociales en el mundo, en el turismo y en el sector hotelero; así como detectar las principales insuficiencias en el uso de las mismas por parte del grupo. 
Determinar el plan de acción que posibilite el

Planificación

mejoramiento de la

promoción de los servicios

y productos del Grupo.

\section{Objetivos específicos.}

- Planificar el uso de las redes sociales de Internet como canal de comunicación promocional del grupo hotelero Gaviota.

- Diversificar los contenidos publicados, a fin de promover el destino Cuba.

\section{Principales acciones de la etapa.}

- Analizar los resultados del diagnóstico elaborado para la conformación de una propuesta de acciones

- Precisar los objetivos, contenidos, métodos, vías, formas, medios y tiempo en que se realizarán las actividades de capacitación.

- Identificar las principales tendencias de los contenidos en las publicaciones

- Diseñar una guía orientadora para las formas de relacionarse con los usuarios. Algunas maneras de relacionarse con los usuarios pueden ser las siguientes: crear y responder preguntas, participar en conversaciones de grupos, publicar contenidos relevantes y llamativos, llevar a cabo desafíos o concursos, valorar los comentarios de los usuarios, entre otras.

- Determinar los principios de la comunicación a través de las redes sociales como: honestidad, humildad, generosidad y respeto.

\section{Descripción de la etapa.}

Para esta etapa se conciben dos momentos: uno dedicado a la planificación de la actividades organizativas y orientadoras para garantizar un eficiente trabajo con las redes sociales; mientras que el segundo momento está dedicado la planificación de los contenidos y el trabajo sobre las plataformas. Como componente rector esta planificación se partirá de determinar los objetivos a mediano, corto y largo plazos, para lo cual se debe tener como ejes el mejoramiento del desempeño profesional de los especialistas de comunicación en el uso de las redes sociales de Internet y herramientas de comunicación online.

Dentro del primer momento también se tienen la configuración del perfil. Una vez determinada las deficiencias, tendencias y redes de interés resultan necesario establecer las características de los perfiles. El perfil debe ser crear desde el lugar del usuario, 
aportándole los atributos más convenientes para lograr una mejor promoción de los hoteles del grupo.

El diseño de la evaluación en cada una de las formas de capacitación a emplear deberá tomar muy en cuenta los objetivos trazados para cada uno de los momentos y el comportamiento de los indicadores que fueron definidos en la operacionalización de dimensiones de la capacitación de los docentes en multimedia educativas. La manera de evaluar puede ser tan diversa como se quiera, pero debe tener un marcado carácter práctico y siempre que sea posible este proceso estará insertado en la práctica profesional del docente.

En la estrategia se propone el uso combinado y coherente de formas de organización para la capacitación. Tres de ellas son: curso de posgrado, talleres y auto-preparación, consideradas e instrumentadas como componentes básicos para el logro de los propósitos de la estrategia; mientras que los cinco restantes: encuentros de intercambio de experiencia, debate, seminarios, consultoría y concurso, son consideradas como complementarias, cuyo rol consiste en apoyar, completar y reforzar las acciones previstas en el diseño para dar cumplimiento y evaluar el desarrollo de los objetivos trazados.

Para el segundo momento se considerarán aquellas acciones en las redes, en este sentido es importante dejar clara las formas de relacionarse con los usuarios.

Ejecución

Establecer las principales pautas de proceder para la promoción de los servicios y productos

\section{Objetivos específicos.}

- Propiciar formas adecuadas para potenciar la promoción de los servicios y productos de alojamiento del grupo hotelero Gaviota

- Ejecutar acciones de control y evaluación durante el trabajo con los perfiles de cada red social de Internet.

- Aumentar el alcance de contenidos y la cantidad de interacciones de los usuarios de los sitios de redes sociales

- Elevar el grado de información del público sobre los productos y servicios de Gaviota

- Responder oportunamente a los comentarios de los usuarios

\section{Principales acciones de la etapa.}


- Diferenciar durante el proceso de promoción los avances de cada uno de los perfiles, ofreciendo los estadísticos necesarios y a su vez la retroalimentación sobre el cumplimiento de los objetivos trazados.

- Evaluar durante el proceso de promoción el cumplimiento de los objetivos propuesto.

- Implementar el seguimiento del estado de cada uno de los perfiles y de sus contenidos.

- Evaluar las respuestas o comentarios. Estructurar un instrumento evaluativo que permita evaluar el impacto de los comentarios

- Propiciar la utilización de hashtags, palabras claves y publicaciones virales.

- Utilizar recursos interactivos que impliquen captar la atención del usurario y motivar el interés sobre un determinado producto.

- Utilizar los seguidores e influencers para que propicien la participación de más usuarios y captar nuevos.

\section{Descripción de la etapa.}

Sobre la base del diagnóstico realizado previamente, se concretan, en esta etapa las acciones planteadas en la planificación, aquí se responde a la interrogante de cómo proceder para emplear de forma eficiente las redes sociales, de forma tal que las instalaciones hoteleras del grupo se promociones de forma efectiva.

Como punto de partida importante en la explicación de esta etapa resulta conveniente destacar que es esta estrategia puede ser instrumentada como parte del sistema de trabajo metodológico. Adecuadamente el carácter flexible con que ha sido diseñada la estrategia, posibilita su ejecución en cada facultad de acuerdo con sus condiciones y características propias, sin alterar la dinámica y el funcionamiento normal de la institución universitaria.

El proceso de promoción será ejecutado en dependencia del nivel donde se esté aplicando la estrategia.

Control y Evaluación.

- Objetivos específicos

- Evaluar de la efectividad de las acciones y operaciones ya ejecutadas con la estrategia propuesta

- Comprobar la efectividad de la estrategia.
Es un sistema de retroalimentación que corrige y orienta las acciones en la dirección adecuada y mantiene latente el proceso de capacitación 


\section{Principales acciones de la etapa.}

- Elaborar evaluaciones de forma sistemáticas, parciales y finales sobre los principales resultados en la promoción de los productos.

- Analizar los datos estadísticos que aportan las redes sociales de Internet.

- Encuestar a los huéspedes sobre las principales vías de obtención de información sobre la marca, y productos del grupo.

- Conocer el grado de satisfacción de los usuarios de las publicaciones.

\section{Descripción de la etapa.}

La misma es ejecutada periódicamente para tomar decisiones indispensables a medida que se vaya desarrollando la estrategia propuesta. El control debe desarrollarse considerando las acciones principales de la segunda etapa.

Una vez ejecutadas las acciones previstas en las etapas anteriores de la estrategia, se hace necesario conocer su efectividad, para lo cual se propone la evaluación de los resultados; por lo tanto, en esta etapa se evalúa la estrategia metodológica propuesta. Este proceso se inicia desde la primera etapa y se mantiene en todo momento, lo que conlleva a la retroalimentación sucesiva de la misma por parte de los actores que participan. Se realiza la evaluación del nivel de satisfacción de las necesidades básicas diagnosticadas.

\section{- Validación de la estrategia.}

Después de elaborada la estrategia se efectuó una consulta a los expertos para comprobar el nivel de aceptación de la misma a partir de la experiencia de empleados del grupo Gaviota. Para ello, se aplicó el método Delphi y los resultados obtenidos consistieron en asegurar la existencia o no concordancia entre los criterios de los expertos. Los resultados estadísticos de la consulta permitieron a los autores considerar los criterios de los expertos en el perfeccionamiento de la estrategia.

El método Delphi está encaminado a obtener las opiniones y criterios de los expertos sobre determinada cuestión. Consiste en la organización de un diálogo anónimo con los expertos consultados individualmente, mediante un cuestionario, con vista a obtener un consenso general. El coeficiente de competencia $\mathrm{K}$ debe estar entre 0,85 y 1 , o sea, $0,85 \leq \mathrm{K} \leq 1$ para que el experto sea seleccionado. En esta investigación, de 34 expertos analizados fueron seleccionados 12 , teniendo en cuenta el coeficiente de competencia a partir de los datos obtenidos en la encuesta aplicada.

Con posterioridad se procedió a la segunda ronda, en la cual se aplicó la primera encuesta dirigida al grupo de expertos seleccionados, con el objetivo de conocer sus criterios y hacer el análisis estadístico de las respuestas en relación a la estrategia propuesta. En la estructura de la encuesta se incluyó la escala de respuesta de tipo Likert con las escalas de valor 
siguiente: muy adecuado, bastante adecuado, adecuado, poco adecuado e inadecuado. De acuerdo a la escala anterior y al comparar los puntos de corte y los valores estadísticos, se obtuvieron los siguientes resultados:

- Del análisis realizado por parte de los expertos acerca de los seis elementos a evaluar, resultó que todos fueron considerados de muy adecuados (MA) para un 100\%.

- En cuanto a la relevancia, la pertinencia y la coherencia de la estrategia propuesta los resultados de las evaluaciones fueron: Muy Relevante y Relevante en el 80\%, Muy Pertinente y Pertinente en el 86,7\% y Muy Coherente y Coherente en el 86,7\%.

\section{Conclusiones.}

El desarrollo y culminación de esta investigación ha permitido arribar a las conclusiones siguientes:

- La primera conclusión que aporta este artículo es que las redes sociales han cambiado las estrategias de comunicación de las empresas y, específicamente, del sector hotelero que está evolucionando hacia nuevos planteamientos y está gestionando su presencia en las redes sociales para buscar sus nichos de mercado. La mayor parte de los hoteles que han colaborado en este estudio reconocen su importancia y también afirman que las usan, probablemente debido a que es relativamente fácil y tiene un bajo coste. Sin embargo, a pesar de la importancia que tienen, es cierto que la gestión de las mismas todavía no es eficiente.

- El referente teórico asumido sobre la estrategia de comunicación de redes sociales de Internet permitió a los autores tomar posturas con respecto al estudio y realizar una propuesta de estrategia que posibilite contribuir a un mejor aprovechamiento de las mismas.

- Las principales redes sociales empleadas por el grupo hotelero Gaviota son: Facebook, YouTube, Twitter y Google Plus. Estas son consideradas como una herramienta de marketing para la promoción de sus productos y marcas.

\section{Referencias bibliográficas.}

Bono, C. d., \& Abad, N. S. (2010). Fundamentos para construir un modelo de comunicación publicitaria desde la perspectiva de la neurociencia. TNS Research, 1-20.

García, G. C., Cortés, P., \& Galmés, M. (2015). Social Media and Communication strategies of social networks in Madrid's hotel industry. Journal of Communication(11), 249282.

Grandi, C. V. (Septiembre de 2013). El uso de las redes sociales como estrategia de marketing en empresas del sector hotelero:una revisión del estado de arte. 
Mena, M. (2013). Innovación en la Promoción Turística. EOI Escuela de Organización Industrial.

Miranda, A. M., \& Cruz, I. (2016). Redes sociales: herramienta de marketing internacional en el sector hotelero. Obtenido de http://www.scielo.org.mx/scielo.php?pid=S187090362016000200012\&script $=$ sci_arttext

OMT. (enero de 2016). Organización Mundial del Turismo. Recuperado el octubre de 2017, de http://www2.unwto.org/es/content/por-que-el-turismo

PCC. (2014). Lineamientos de la Política Económica y Social del Partido y la Revolución. La Habana: Congreso de la República de Cuba.

Ruiz Cora, D. (2018). El uso de las Redes Sociales en los grupos hoteleros Cubanacán Gaviota e Islazul. La Habana.

SGM, S. G. (2016). Marketing a la medida de tus necesidades. Obtenido de file://F:/daniela\%20tesis/8.\%20Tesis\%20Daniela\%20redes/8.\%20Tesis\%20Daniela /Estrategia\%20de\%20redes\%20sociales\%20para\%20hoteles\%20\%20Marketing\%20SGM.htm

Villasante, T. (2010). Redes sociales para la investigación participativa. Sociedad de $\operatorname{Hoy}(18), 109-129$.

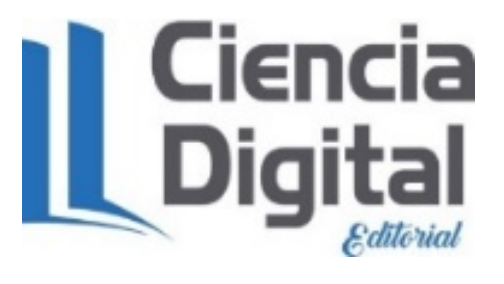




\section{Para citar el artículo indexado}

Pulido de la Paz, D., \& Vázquez Alfonso, Y. (2020). Propuesta de una estrategia de comunicación para la empresa hotelera Gaviota. AlfaPublicaciones, 1(2), 52-67. https://doi.org/10.33262/ap.v1i2.6

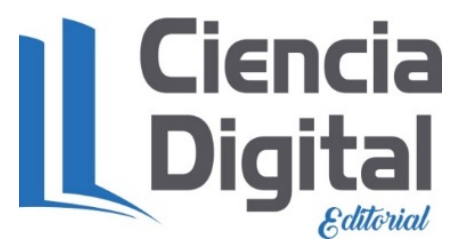

El artículo que se publica es de exclusiva responsabilidad de los autores y no necesariamente reflejan el pensamiento de la Revista Alpha Publicaciones.

El artículo queda en propiedad de la revista y, por tanto, su publicación parcial y/o total en otro medio tiene que ser autorizado por el director de la Revista Alpha Publicaciones.
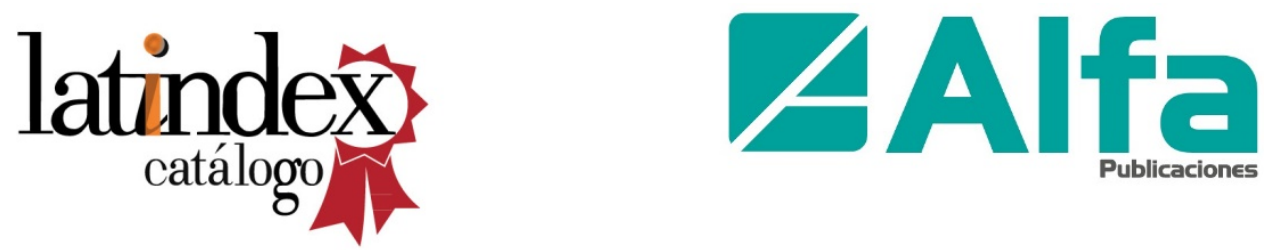